Vierteljahrsschrift für Geschichte der Medizin und der Naturwissenschaften Revue trimestrielle d'histoire de la médecine

\section{GESNERUS}

Jahrgang/Vol. 271970

Heft/Fasc. $1 / 2$

\title{
Geschichtliches zur Lehre von der Automatie, Unterhaltung und Regelung der Herztätigkeit*
}

Von K.E.Rothschuh, Münster (Westfalen)

\section{Einleitung}

Jedes Zeitalter hat seine Art, Phänomene zu sehen, daran Fragen anzuknüpfen, sie zu lösen und sie zu interpretieren. Das heißt: verschiedene Epochen der Geschichte lassen deutlich verschiedene Sehweisen und Interpretationstendenzen erkennen ${ }^{1}$. In unserer Zeit ist man in weit höherem Maße als in anderen Zeiten bestrebt, Ursachen -Wirkungs - Zusammenhänge aufzuklären, sie quantitativ zu erfassen und aus den aufgefundenen Zusammenhängen geeignete Teilstücke wieder so zu kombinieren, daß die so analysierte Natur in den Dienst menschlicher Zwecke gestellt werden kann. Auf das Lebendige angewandt, heißt das: der Physiologe analysiert die Lebensvorgänge, und der Mediziner lernt daraus, die Lebensvorgänge zu manipulieren. Diese Sehweise der Natur ist nicht die einzig mögliche. Man kann die Natur auch befragen auf ihre wunderbare Ordnung und die Ergebnisse in den Dienst der Gottesverehrung stellen. Oder man kann durch Analogieschlüsse und generalisierende Verallgemeinerung von Beobachtungen ein philosophisches Gesamtsystem der Natur entwerfen, welches

* Als Dr.-Markus-Guggenheim-Vortrag gehalten an der Jahresversammlung der Schweizerischen Gesellschaft für Geschichte der Medizin und der Naturwissenschaften, St. Gallen, den 4. Oktober 1969.

1 Vgl. dazu K.E.Rothschun, Physiologie, Der Wandel ihrer Konzepte, Probleme und Methoden vom 16. bis 20. Jahrhundert [= Orbis Academicus, Band II/15] Freiburg/ München 1968. 
die Rolle, den Sinn des Einzelnen in einem Ganzen angibt und mit diesem Verständnis zwar Befriedigung, aber keine Macht verleiht. Unsere Zeit beschäftigt sich sehr viel weniger mit den Fragen der Lebensordnung (Bionomie) als mit der Lebenstechnik (Biotechnik) und ihren Mechanismen ${ }^{2}$, und von dieser Sicht soll in bezug auf das Herz mit besonderer Berücksichtigung seiner Automatie und der Regelung seiner Tätigkeit die Rede sein. Wir können dabei an Fragestellungen unserer technischen Welt anknüpfen.

In vielen technischen Anordnungen ist die «Selbsttätigkeit» ein hervorragendes Merkmal, zugleich die Beeinflußbarkeit ihrer Prozesse durch Steuerungsanweisungen und den Einbau von informationsverwertenden Regelungsschaltungen. Das regt wieder an, solche Modelle auf das Lebendige zu übertragen. Denn die Spontaneität, die Selbsttätigkeit, die Steuerung und Regelung sind auch Grunderscheinungen alles Lebendigen. Die Biokybernetik, die sich damit beschäftigt, ist heute ein riesiges Forschungsgebiet geworden, welches mit neuen Begriffen und der Technik entlehnten Denkmodellen gewisse Leistungen des Organismus besser als bisher verständlich zu machen sucht ${ }^{3}$. Das Herz bietet hier ganz besondere Probleme. Das Herz ist der Prototyp des dauernd tätigen Organs. Mit dem ersten Herzschlag beginnt das Leben, mit dem letzten hört es auf ${ }^{4}$, sagt Aristoteles. Was veranlaßt seine Tätigkeit, wie und wodurch vollzieht sich die Schlagfolge der Herzabteilungen, was verändert die Herztätigkeit, wenn wir uns körperlich anstrengen oder erregt sind ? Seine Spontaneität, die sich ein Leben lang erhaltende Aktivität, die pausenlose Prozessualität, das geordnete Zusammenwirken der vielen Glieder des Herzens haben Naturphilosophen, Ärzte und Physiologen seit Jahrtausenden immer wieder zu Erklärungsversuchen herausgefordert. Sie benutzten andere Begriffssysteme als heute, aber ihr Anliegen war letzten Endes das gleiche wie das unsrige. Davon soll heute die Rede sein, also nicht von der Geschichte der Herzlehre insgesamt, sondern vor allem von der Geschichte der Herzautomatie und Selbstregelung.

2 Vgl. dazu K.Е. RотнsснUн, Theorie des Organismus, 2. Auflage, München 1963.

3 Vgl. Volker Henne, Materialien zur Vorgeschichte der Kybernetik, Studium Generale 22 (1969) 164-190. - H.ScHMIDT, Regelungstechnik, vdI-Zeitschrift 85 (1941) 81-88. Norbert Wiener, Cybernetics, Paris/New York 1948.

${ }^{4}$ Carl Wilh. Sievert, Die Physiologie des Aristoteles, Diss. Med., Münster 1948 (Maschinenschrift). 


\section{Das Herz als Sitz der Seele und der eingeborenen Wärme}

Der erste große Lehrmeister der Biologie in der Antike, Aristoteles, setzt das Herz absolut in das Zentrum des Organismus. Der Leib ist ein beseelter Stoff, denn «alles Lebendige ist beseelt», und jedes Glied unseres Körpers ist ein zweckmäßiges Werkzeug im Dienste des Lebens und der Seele $^{5}$. Das Herz ist auch der Sitz der Seele und des Sensorium commune ${ }^{6}$. Das Herz ist auch der Ort der Blutbereitung durch die dort wirkende eingeborene und lebenspendende Wärme. Die Herzbewegung ist eine Folge von Erwärmung und Abkühlung des Blutes. Der Pulsschlag entsteht im Herzen und erfolgt ${ }^{7}$ durch ein Heben der Herzwände durch eine wärmebedingte Volumenzunahme der Flüssigkeit im Herzen. Durch die Atmung und die Abkühlung mildert sich die Wärme, und deshalb sinkt das Herz wieder zusammen. Aristoteles lehrte also eine ausgesprochen kardiozentrische Organismuslehre im Rahmen seiner gesamten Naturphilosophie.

Wie kommt aber das Herz an seine Fähigkeit zu schlagen? Darauf gibt Galenos (etwa 129-201 n.Chr.) Antwort. Im lebendigen Körper gibt es zweckmäßig wirkende Kräfte (dynameis, facultates), und zwar animale, vitale und natürliche Kräfte. Das Herz ist Sitz der vitalen Kräfte, dazu gehört erstens die facultas pulsifica, die auch den Arterien zukommt, also eine Kraft der Zusammenziehung und Ausdehnung, die sich ohne Reiz von selbst vollzieht. Zweitens gehört zum Herzen die eingeborene Wärme (Calor innatus), die zur Anreicherung des arteriellen Blutes mit vitalen, heißen, belebenden Spiritus dient ${ }^{8}$. Eine Kreislaufvorstellung hatte GaLEN noch nicht, wohl eine Blutverteilungslehre ${ }^{9}$. Dabei hat das Herz ein in der Diastole wirkendes anziehendes Vermögen. Das ist die eigentlich bewegende Kraft für den Blutfluß zum Herzen und für die Blutaufnahme in den

5 Aristoteles, Von Jugend und Alter, Leben und Tod, Cap. 6; ferner in Von der Seele, 2. Buch.

6 Aristoteles, Von Jugend ..., Cap. 3.

7 Aristoteles, Vom Atmen, Cap. 20.

${ }^{8}$ Galen behauptet: Wenn man bei eröffnetem Thorax in die linke Herzkammer eines Tieres einen Finger einführt, «invenies enim locum hunc omnibus quae in animali sunt, haud paulo calidiorem » (De temperamentis, Liber II, in KüHN, Galeni Opera, Tom. I, Lipsiae 1821, S. 601).

9 Vgl. R.E.Siegel, Galen's system of physiology and medicine, Basel/New York, Band I, 1968. - К.Е. Rотнsснuн, Geschichte der Physiologie, Berlin 1952. 
Organen ${ }^{10}$. Der Auswurf des Blutes durch die Systole tritt demgegenüber zurück. Die Längsfasern erweitern bei ihrer Zusammenziehung die Herzräume, die Querfasern verengen sie $^{11}$. Das Herz wird also kaum als Pumpe aufgefaßt, denn die Blutmenge ist klein und in den Arterien eher schaumig als wässerig flüssig.

Im 13. Jahrhundert begegnet uns in Thomas von Aquin ein Denker, der Aristotelisches und Christliches vereinigt. In einer Schrift De motu cordis ${ }^{12}$ stellt er die Frage nach der Rolle des Herzens. Alles Bewegte, so sagt Aristoteles, bedarf eines Bewegers. Die Bewegung in den Lebewesen beruht nicht auf äußeren Ursachen, sondern auf einem inneren Prinzip, und dies ist die Seele. Das gilt auch für das Herz. Diese Tradition greift in der Mitte des 16. Jahrhunderts der überragende Pariser Arzt Jean Fernel $(\dagger 1558)$ wieder auf. Er vereinigt Elemente der aristotelischen, galenischen, arabischen und thomistischen Physiologie. Im Mittelpunkt seiner Physiologia steht nicht das Herz, sondern die Seele. Sie beseelt und belebt den Körper ${ }^{13}$, der ihr von Gott zum Dienste geschaffen und zum vorübergehenden Wohnsitz angewiesen wurde. In diesem Hause waltet die Seele und bedient sich der Organe und Funktionen als ihrer Instrumente. Die anima besitzt dazu die drei schon erwähnten Vermögen, die facultas animalis, die facultas vitalis und die facultas naturalis. Sie sind Anteile der Seele und Kräfte, die von der Seele ausgehen. Die Seele ist also die erste Ursache, die Vermögen sind helfende Ursachen. Sie lenken und leiten. Um auf den Körper wirken zu können, bedarf die immaterielle Seele mit ihren Vermögen der vermittelnden Medien zur Materie des Körpers, eines ätherischen Spiritus animalis für die Betätigung der Bewegung und Empfindungsorgane, einen luftigen Spiritus vitalis für die Übertragung von Belebung und Erwärmung vom Herzen in die Peripherie, und einen dampfartigen Spiritus naturalis zur Vermittlung von Erzeugung, Ernährung und Wachstum (Abb. 1). Alle Zweckmäßigkeit geht also von der Seele aus, auch der

10 Galen: «majore enim vi cor dilatatum attrahat (!) necesse est, quam contractum expellat», De usu partium corporis humani, Liber VI, Cap. 15, in KÜHN, Tom. III, S. 484.

11 Galen, ebenda, Liber VI, Cap. 8 (KüHN, Tom. III, S. 439).

12 Thomas von Aquin, De motu cordis (ca. 1270); vgl. englische Übersetzung und Diskussion von V. R. Larkin, St. Thomas Aquinas on the Movement of the Heart, J.Hist. Med. Allied Sci. 15 (1965) 22-30.

13 Vgl. K. E. RothschuH, Das System der Physiologie von Jean Fernel (154.2) und seine Wurzeln, Verhandlungen des 19. Internationalen Kongresses der Geschichte der Medizin, Basel 1964, S. 529-536, Basel/New York 1966. 


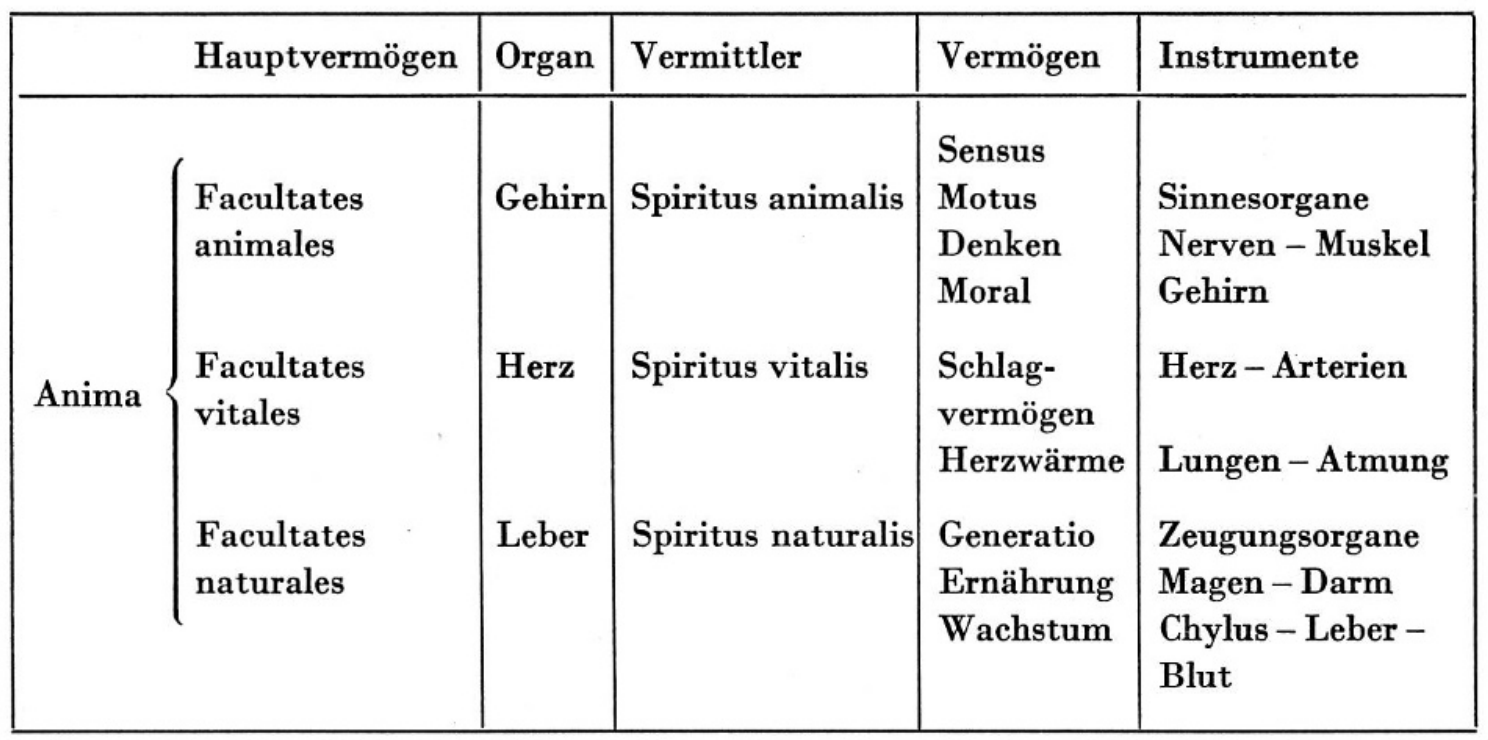

Abb. 1. Die Lehre von den Facultates bei Fernel 1542

Herzschlag. Nun gibt es im Körper viele anatomisch bekannte Organe, und Fernel schildert, was sie als Instrumente der Anima leisten. Das Herz hat gemäß GALEN eine vis pulsifica. Es zieht Blut und Lungenluft an und stößt erwärmtes und mit Spiritus versehenes Blut in die Arterien hinaus. FerneL lehrt wenig Neues, aber er verarbeitet und systematisiert die gesamte traditionelle Physiologie als Abschluß der bisherigen Denkarbeit. Seine Organismuslehre war viel mehr spiritualistisch als anatomisch oder mechanisch unterbaut. Ein wirkliches Ernstnehmen der anatomischen Details beginnt erst seit der Mitte des 16. Jahrhunderts und führt dann schnell zu einer ganzen Anzahl wesentlicher Korrekturen an der traditionellen Physiologie ${ }^{14}$.

\section{Das thermodynamische kardiozentrische System des Descartes}

Noch bei Theodor Zwinger (1610) bewegt die Respiration stärker das Blut als das Herz selbst ${ }^{15}$. Und bei Daniel Sennert (1626) ist noch mehr

14 Vgl. K. E. RothschuH, vgl. Anm. 1, S. 50-59.

15 Theodori Zwingeri, Physiologia Medica, Basileae 1610, Cap. XVII, S. 337: «Das Blut der Arterien bewegt sich nicht wie das Wasser oder das Blut in den Venen, sondern nach Art des Feuers. Es hat eine natürliche Bewegung durch die Lungen, die das Blut, das wegen seiner Feinheit und Siedung sehr dazu geneigt ist, nicht anders bewegen als die Winde das Meer zu bewegen pflegen.» 
von der Dilatation und Ansaugung des Blutes durch das Herz die Rede ${ }^{16}$ als von der Blutaustreibung. An eine Pumpenmechanik wird also bis dahin kaum ernsthaft gedacht.

Im Jahre 1628 erschien das Buch von William Harvey ( $\dagger$ 1657), in dem er seine Beobachtungen und Experimente mitteilt, die ihn veranlaßten, die Blutbewegungslehre des Galenos und Fernel aufzugeben und eine Kreisbewegung des Blutes zu behaupten. Harvey bewies, daß das Herz mit seiner rechten Kammer das Blut durch die Lunge zur linken Kammer schickt. Von dort wird es über die Arterien über die Kapillaren auf einen Kreisweg durch den Körper getrieben. Aber wozu das Ganze? Das Blut wird in den Organen abgekühlt, verdichtet und geschwächt. Daher muß es zum Herzen zurück, um wieder seine Vollkommenheit zu erlangen. «Dort wird es durch die natürliche kräftige feurige Wärme, diesen Lebensschatz, von neuem verflüssigt, mit Spiritus und sozusagen mit Balsam angereichert und verteilt» (Cap. 8). Das Herz ist das erste im Fötus gebildete Organ, eine Art innerlichen Lebewesens, von ihm hängt alles ab (Cap. 17). Harvey hat als erster die Arbeitsweise der einzelnen Herzabteilungen und ihre Folge richtig beschrieben. Die Fähigkeit des Schlagens bleibt auch bei ihm ein ursprüngliches Vermögen, das nicht erklärt werden kann. Er hat auch nicht erklärt, wodurch oder warum die einzelnen Herzabteilungen sich in einer ganz bestimmten Folge nacheinander kontrahieren. Wie AristoTELES und GALEN hält HARVEY 1628 noch an der eingeborenen Wärme des Herzens fest. Diese Überzeugung war damals stärker als jede sinnliche Beobachtung. JAN DE WALE (1641) ${ }^{17}$ und Colombo (1659) ${ }^{18}$ steckten einen Finger in ein freigelegtes und der Spitze beraubtes Tierherz und empfanden eine kaum erträgliche Hitze. Ja HARveY sieht 1651 im wenige Tage bebrüteten Hühnerei bei jeder Systole des ersten Bluttropfens einen kleinen Funken aufblitzen ${ }^{19}$ als erstes Aufleuchten der flammula cordis ${ }^{20}$. Bei

16 Danieli Sennerti, Institutiones Medicinae, 3. Editio, Wittebergae 1628, Liber III, S. 614: «Dilatatur autem cor a peculiari facultate sibi insita, quam vitalem et pulsificam nominant. » Aus dieser Stelle ist ersichtlich, wie wenig mechanisch die ganze Blutbewegung noch zur Zeit Harveys gedacht wurde.

17 JAN DE WALE, Epistulae duae de motu chyli et sanguinis ad Th. Bartholinum I. und II. Als Anhang zu Bartholins Institutiones Anatomicae, Lugd. Bat. 1641.

18 Realdo Colombo, De re anatomica, Libri XV, 1559.

19 HARVEY schrieb in: De generatione animalium, 1651, Cap. 17: «Iam enim colliquamenti limbus linea exibi sanguinea purpurascens rutilat: ejusque in centro fere, punctum sanguineum saliens emicat; exiguum, adeo ut in sua diastole, ceu minima ignis scintillula effulgat, et mox in systole visum prorsus effugiat et dispareat » (4.Tag). 
HaRvey treibt die systolische Kontraktion das Blut dureh den ganzen Kreislauf. Bei Hermann Conring (1643) wird das Blut teils vom Herzen angezogen, teils ausgetrieben. Bei der Anziehung spielt die verschiedene Wärme der Herzabteilungen eine wichtige Rolle. Je größer die Wärme einer Herzabteilung, desto stärker zieht sie das Blut an, es fließt von den kühleren Eingeweiden zur wärmeren Leber, von dort zum wärmeren rechten Vorhof usw. bis zur besonders heißen linken Kammer: Damit das arterielle Blut aber zu den kühleren Organen gelangt, muß es vom Herzen durch Pulsio, Vectio und Vertigo ausgetrieben werden ${ }^{21}$.

Um 1630 beginnt René Descartes (†1650) ein neues Bild von Natur und Mensch zu erarbeiten, welches einen radikalen Bruch mit der gesamten Tradition bedeutete ${ }^{22}$. Er scheidet die Welt in einen ausgedehnten Anteil (mit Materie und Bewegung) und in einen denkenden Anteil (d. i. die denkende, empfindende und wollende Seele). Die Materie, aus kleinsten Partikeln verschiedener Gestalt und Größe bestehend, befindet sich ständig in Bewegung. Je stärker diese ist, je mehr eine Materie an schnellbewegten Teilchen besitzt, desto wärmer ist sie. Diese Wärme ist nicht nur die wichtigste Bewegungsquelle im ganzen Kosmos, sondern auch im Organismus. Denn auch der Organismus gehört zur res extensa, in der alles zwangsläufig ohne die Mitwirkung seelischer Kräfte erfolgt. In mehreren Schriften (1630 und 1648) hat Descartes ${ }^{23}$ die Grundzüge einer radikal veränderten, durch materia und motus bestimmten Physiologie entworfen, in der das Herz wieder eine zentrale Rolle spielt, aber auf völlig anderer Basis. Dessen Blutauswurffunktion inmitten des Kreislaufs übernahm er von HARvey, aber die Natur, Ursache und Energiequelle der Herzbewegung sah er völlig anders. Die Gründe von Descartes für diese andere Erklärung waren folgende:

20 Noch 1667 heißt es bei Thomas Bartholinus in De flammula cordis epistula cum Jacobi Halsti v. cl. ejusdem argumenti dissertatione, Hafniae (S. 4) «Si tam patulum oculis nostris esset pectus animalium, quam cicindulae transparens corpusculum, forsan non absimile lumen in corde appareret, quod extinguitur, quam primum externo aeri patet.» Etwas später (S. 6) heißt es: «de interna cordis flammula, in qua vitae nostrae ratio consistit.»

21 Hermannus Conringius, De sanguinis generatione et motu naturali Opus novum, Helmstadii 1643, Cap. 42 , S. 335.

${ }^{22}$ K.E. Rothschun, René Descartes und die Theorie der Lebenserscheinungen, Sudhoffs Arch. 50 (1966) 25-42.

${ }^{23}$ Descartes, L'Homme (1630-32), und La description du corps humain (1648). Beide Schriften erschienen posthum. Die erste deutsche Übersetzung durch K. E. RothschUH erschien kürzlich im Verlag Lambert Schneider, Heidelberg. 
Hat die Seele als immaterielles Prinzip auf materielle Körper keinen Einfluß, dann kann es keine wirkenden facultates für die Herzbewegung, die Muskelbetätigung, die Verdauung und Absonderung geben, sondern alle Vorgänge in den Organen müssen durch ein naturgesetzliches Kontinuum mechanischer Bewegungsvorgänge unter materiellen Teilchen erklärt werden; nicht anders wie man es von den Automaten kennt, die von dem Wasser in den königlichen Gärten von Versailles in Betrieb gesetzt werden. Dort genügt der Druck des Wassers, um z.B. Maschinen Musik machen zu lassen, um Statuen Worte sprechen zu lassen und zu veranlassen, daß eine Dianafigur sich vor einem Besucher im Schilf versteckt ${ }^{24}$. Könnte nicht Gott, so meint Descartes, auch in den Organismus des Menschen alle jene Teile hineingelegt haben, die analog zu Maschinen bewirken, daß er gehen, essen, atmen usw. kann ${ }^{25}$ ? So entwirft Descartes ein Bild der Menschmaschine, in der alles nach mechanischen Prinzipien funktioniert. Das Herz ist der Antriebsmotor dieser Maschine, eine Art Wärmekraftmaschine. Denn der Blutauswurf aus dem Herzen erfolgt nicht durch ein Kontraktionsvermögen des Herzmuskels, sondern dadurch, daß das in die Kammern einfließende Blut durch einen hitzeentwickelnden Prozeß der Fermentation nach Art von Milch ${ }^{26}$ zum Aufwallen und zur Ausdehnung gebracht wird. Das treibt das mit allen Partikeln stark bewegte Blut in die großen Arterien hinaus, die es in den Körper verteilen. Abgekühlt und weniger bewegt, gelangt es wieder zum Herzen zurück. In den Kammern trifft es wieder auf die erwähnte, nicht leuchtende Quelle der Wärme und Fermentation; das Blut dehnt sich erneut aus (Abb. 2) usw. Je nach der Struktur der Organe, der Muskeln, der Drüsen und der Nerven vollziehen die bewegten Blutteilchen bestimmte physiologische Leistungen, alles ohne die immateriellen Facultates des GaLEN und des FERNEL, und rein mechanisch. So gesehen, sagt Descartes, haben wir keinerlei Anlaß zu der Annahme, $\mathrm{da} \beta$ es unsere Seele ist, welche in der Menschmaschine die Bewegungen in den Organen hervorruft, so wenig wie wir annehmen müssen, daß es in der Uhr eine Seele gibt, welche die Stunden anzeigt ${ }^{27}$. Das Herz bewegt also nicht das Blut, sondern das bewegte erhitzte Blut bewegt das passive Herz.

${ }^{24}$ Descartes in der Übersetzung von K. E. RothschUH, S. 57.

25 Ebenda, S. 135.

${ }^{26}$ Dieser Vergleich mit der Milch stammt von Primirosius, Exercitationes et animadversiones de motu cordis et circulatione sanguinis, Londini 1630, Cap. IX, S. $71 \mathrm{ff}$.

${ }^{27}$ Descartes in der Übersetzung von K. E. RothschUH, S.141. 


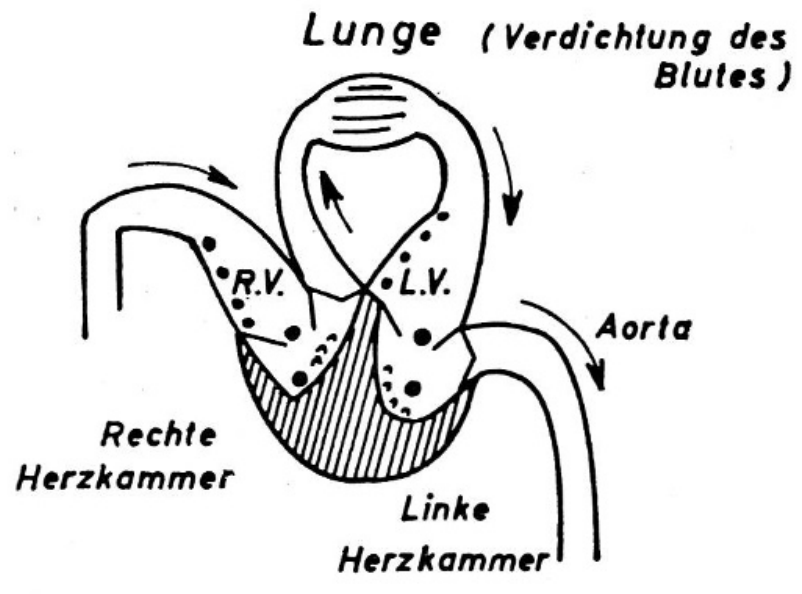

Systole

Kammerfüllung mit abgekühltem verdichtetem Blut

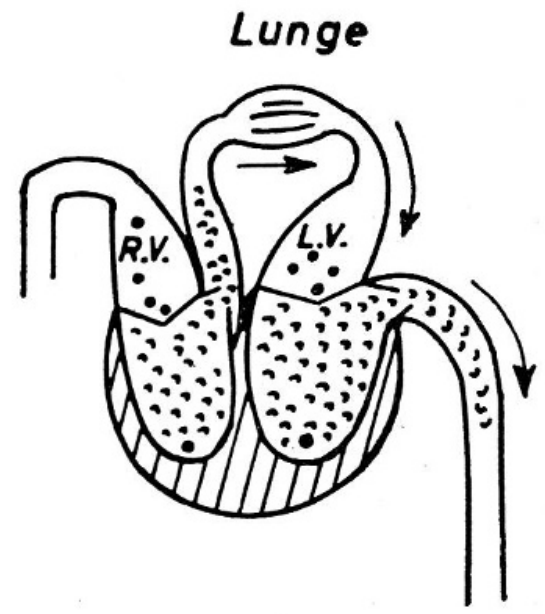

Diastole

Kammerentleerung durch Aufwallen des Blutes

Abb. 2. Herzaktion bei Descartes 1648 (Schema)

So wird die vis pulsifica überflüssig. Die Herztätigkeit steuert sich selbst durch einen Wechsel von Blutausdehnung und Blutverdichtung. Die Koordination und Lenkung der Herzfunktion erfolgt durch die anatomische Folge der Herzabteilungen, der Gefäße und durch die Anordnung der Klappenventile. So entwarf Descartes das erste rein technomorphe Modell des Organismus auf kardiothermodynamischer Grundlage. Damit nicht genug. Descartes beschrieb auch die Wege, auf denen sich eine solche Menschmaschine aus Samen und mütterlichem Blut von selbst erzeugen und bilden kann. Damit wurde auch dieser Prozeß von der Hypothese einer bildenden, mit Intelligenz und Zwecktätigkeit begabten seelischen Potenz abgelöst - mit äußerster Konsequenz! Descartes' kühne Vorstellung von der Herztätigkeit hat seinerzeit überraschend viele Anhänger gefunden, z.B. Henricus Regius ${ }^{28}$, Theodor Craanen ${ }^{29}$, Franciscus Syluius ${ }^{30}$,

28 Henricus Regius, Physiologia sive cognitio sanitatis, Ultrajecti 1641.

29 Theodor Crannen, Tractatus Physico-Medicus, Diss. Physico-Medica de Homine, Leyden 1689.

30 Franciscus Sylvius vertritt in Disputationum Medicarum Decas, Nr. III, De Chylis... $\S \S X V$, XVI, XXX die Cartesische Herzlehre. Er rückt in «de febribus» wieder davon ab. 
Cornelis Bontekoe ${ }^{31}$, Cornelis van Hoghelande ${ }^{32}$ und a.m. Schließlich sei erwähnt, daß Descartes ausdrücklich die Regelung des Blutauswurfs und -einflusses durch einen Herznerven erwähnt, und wenn man seine Darstellung der Gehirnfunktionen liest, wird man dort auch auf die Idee der Rückkoppelung stoßen ${ }^{33}$.

Was aber wendet der Anatom Thomas Bartholinus 1641 u.a. gegen Descartes ein ? Es wäre gegen die Würde des Prinzipalorgans Herz, wenn es von etwas anderem wider Willen und mit Gewalt in Bewegung gesetzt würde ${ }^{34}$. Daraus spricht noch einmal jene außerordentliche Bewertung des Herzens ${ }^{35}$ im 17. Jahrhundert, wie sie etwa JEAN Riolan (1626) erkennen läßt. Wenn nicht alles täuscht, steht auch die Entstehung des Herz-JesuKultes in diesem Jahrhundert mit dieser Haltung in Zusammenhang ${ }^{36}$. Erst 1664 hat ausgerechnet Niels Stensen, der spätere katholische Priester, als Anatom die Muskelnatur des Herzens aufgeklärt und den Mythos vom Herzen als Sitz eines Feuers, als Thron der Seele und Ursprung der belebenden Kraft der vitalen Spiritus zurückgewiesen und gesagt ${ }^{37}$ :

31 Connelis Bontekoe, Fundamenta Medica sive de Alcali et Acidi effectibus ..., Amstelodami 1688. Auch Johannes Clauberg, Opera Physica, Amstelodami 1664 (S. 315) gehört hierher.

32 Cornelis van Hoghelande, Cogitationes, quibus dei existentia et animae spiritalitas et possibilis cum corpore unio, demonstrantur ..., Amstelodami 1646.

33 Descartes in der Übersetzung von K. E. RотнschUH, S. 99, $114 \mathrm{ff}$.

34 Thomas Bartholinus, in Caspari Bartholini, Institutiones Anatomicae, Lugd. Batav. 1641, S. 206: «esset contra majestatem partis principis ab alio se invito moveri et impressionem violentam recipere. $\gg$

35 Joanni Riolani, Anthropographia, Parisiis 1626 (S. 360): "Expeditis et absolutis pulmonibus ad cor ipsum accedes, nobilissimam totius corporis partem, irascibilis animae sedem, facultatis et spiritus vitalis fontem, caloris nativi focum, atque ut uno verbo dicam, nostri corporis Solem, cujus influxu omnia viscera calent et recreantur. »

${ }^{36}$ Seit der Hl. Margareta M. Alacoque $\left({ }^{*} 1647\right)$ wird die Herz-Jesu-Verehrung in das öffentliche Gebet der Kirche aufgenommen; das erste Herz-Jesu-Fest wurde 1686 gefeiert. Besonders beteiligt waren die Jesuiten. Vgl.Jos. DE GallifFet, S. J. De cultu sacrosancti Cordis Dei ac Domini Nostri Jesu Christi, 1685. Nach Josef StIERLI, Cor salvatoris, Wege zur Herz-Jesu-Verehrung, Herder, Freiburg 1954. Aber die Herz-Jesu-Verehrung war auch nicht neu, denn als Teresa de Jesus (1515-1582), eine Karmeliterin, heiliggesprochen wurde, erhielt sie das brennende Herz als Attribut (vgl. KarL RichstaEtTER, Die Herz-Jesu-Verehrung im deutschen Mittelalter, 2. Auflage, München 1924, und Monus, Eine Weltgeschichte des Herzens, Hamburg 1959, S. 122 ff.).

37 Nicolai Stenonis, De musculis et glandulis observationum specimen, 1664 [nach Bibliotheca Anatomica, Ed. D. Le Clerc et J.Mangetus, Tom. II, Genevae 1685, S. 532]. 
«Soweit ich solche Dinge beurteilen kann, fürchte ich, daß alle Argumente über die Würde der Körperteile Redensarten sind.» Aber bis heute blieb das Herz Symbol für Gemüt und Charakter, noch immer bricht das Herz vor Kummer und hüpft es vor Freude, noch immer gibt es ein heißes Herz und ein hartes, kaltes Herz.

\section{Chemische Mechanismen bei der Herztätigkeit}

Descartes läßt - wie gesagt - bei der Ausdehnung des Blutes im Herzen eine «Fermentation» mitspielen. Fermentation ist hier Erzeugung einer inneren Bewegung der Teilchen, aus denen das Blut besteht. Diese Bewertung der Fermentation geht wohl u. a. auf SÉBASTIEN BAsso zurück, der um 1620 eine neoatomistische Lehre von den chemischen Prozessen entwickelt. In allen Körpern findet man Spiritus, Oleum, Phlegma, Sal und Rückstand, wenn man sie der Destillation unterwirft. Wohl über Gassendi, den Neoatomisten in Frankreich, kam die Lehre auch zu dem Arzt Tномаs WiLlis in Oxford. Er schloß sich in der Erklärung der Lebensvorgänge den Atomisten an. Sein wichtigster erster Traktat behandelt die Fermentatio (1659). Er erklärt sie als innere Bewegung, die auf der Wirkung von öligschwefligen Teilchen beruht und nichts anderes als Wärme ist. Die Spiritus des Blutes sind allerfeinste Teilchen, die durch Fermentation entstehen und das Blut in eine dauernde innere Bewegung versetzen. Sie ist am stärksten im Herzen und bringt das Blut dort zur Aufwallung und zum Ausfließen ${ }^{38}$. $\mathrm{Ja}$, der Zustand des Lebens beruht eigentlich auf diesem Motus intestinus des Blutes ${ }^{39}$. Anhänger der Fermentationslehre war auch Јон. ВАPтIST van Helmont (1648) in den Niederlanden, der dem «Fermentum » in allen seinen Abarten und in allen Organen die eigentlich aktivierende, lenkende Rolle im Organismus, ja die Bildungsvorgänge im Keim zuschrieb. Auch im Herzen, und zwar in der linken Herzkammer, wohnt eine Gärungskraft, welche das Blut in allerfeinsten Spiritus verwandelt, der einem subtilen Äther gleicht. Diese korpuskulare Theorie der Materie und der chemischen Prozesse ist eine Art von Physiko-Chemie. Die fermentativen Veränderungen sind nicht nur Quelle neuer stofflicher Eigenschaften, sondern auch Ursache für Bewegung und Wärme. Auf ihr beruht alles Leben und jede Tätigkeit der Organe. Diese scheinbar vom Thema abliegenden Dinge mußte

38 Thomae Willis, Opera Omnia, Amstelodami 1682, darin: De Fermentatione; besonders S. 11/12.

39 Thомae WiLlis, ebenda: De febribus; besonders S. 52. 
ich hier erwähnen, weil die Verbindung von Korpuskularlehre und Biophysik wieder zu neuen Theorien der Herzautomatie und Herzaktivität geführt hat. Insbesondere lebte die Lehre wieder auf, daß die erste Lebensregung im Keim nicht im Herzen, sondern durch eine Fermentation im Blute beginnt, woraus wieder folgt, daß dann die Herzbewegung eine Folge der Blutbewegung und nicht ihre Ursache sei ${ }^{40}$.

\section{Die Herznerven als Quelle der Herztätigkeit}

Eine neue Interpretation für die Ursache der Herzautomatie entsteht im 17. Jahrhundert mit der Lehre von den Herznerven. Genau vor 300 Jahren, im Jahre 1669, erschien das Buch De corde von Richard Lower, einem Mitarbeiter von WiLlis und hervorragendem Experimentator. Er leugnet wie Stensen, daß im Herzen eine größere Wärme existiere als anderswo im Körper. Auch verwirft er die cartesische Ebullitio, die Aufwallung als Ursache der Herzbewegung ${ }^{41}$, denn noch kleinste Stücke der Herzmuskulatur fahren fort zu schlagen. Auch gäbe es im Herzen überhaupt keine Fermentatio, keine innere Blutbewegung. Zwei Dinge löschen die Herzaktion schnell aus, der Mangel an Luft und die Durchtrennung der Herznerven. Lower behauptet, daß vom Kleinhirn durch die Herznerven ununterbrochen ein flüssiger spirituöser Nervensaft herzwärts fließt und dort den Herzschlag hervorruft ${ }^{42}$. Wie er das macht, das zu wissen, sei Gottes Vor-

40 Vgl. Walter Pagel, William Harveys Biological Ideas, selected aspects and historical background, Basel/New York 1967.

41 R. Lower, De corde (1669), nach der Ausgabe in Bibliotheca Anatomica, Ed. D. LE Clerc et J.Mangetus, Tom. II, 1685, S. 93.

42 Die Vorstellung, daß der Herzschlag von den Herznerven herrührt, findet sich schon in Tномas Willis' Cerebri Anatome (1664, Cap. 24). Es heißt dort (nach Opera Omnia, Ed. Blasius, Amstelodami 1682, S. 86/87): Das Herz ist mehr ein Muskel als ein Parenchym, es erhält über die Nerven des achten Paares «motus sive actionis instinctus.» Durchschneidet man die Herznerven beim lebenden Hund, so wird des Herzens «motus piger et impotens». Wenn die gesamten Herznerven «penitus dissecarentur, statim extinguebitur». Ohne Nerven und ohne Blut "potentia motiva adimit». Über diese Versuche (wohl mit LowER, welchen WiLlis im Vorwort erwähnt) berichtet auch LOWER in De Corde, 1669. Er sagt über das Zusammenwirken von Gehirn und Herz: Das Herz treibt das Blut ins Gehirn «et spiritus vice versa per nervos in cor influentes motum ejus perennem et constantem vicissim conservant: adeo ut cordi debeatur, quod spiritus in cerebro nusquam deficiant, quin et cerebrum hoc agit ut cordis motus nusquam cesset» (Bibl. Anat. l.c., Anm. 41), S. 96. Auch Johannes Bohn, Exercitationes Physiologicae, Nona 1671, Leipzig, schließt sich WILLIS und LowER an. 
recht. Jedenfalls bestehe eine Zusammenarbeit zwischen Herz und Gehirn, denn das Herz schicke das nötige Blut ins Kleinhirn, und dieses liefert daraufhin wieder die Spiritus für die Herzaktion: das ist ein «mutuum subsidium et ministerium» (Cap. 96), eine Art von Rückkopplung in heutiger Terminologie. Fließt mehr Blut aus den arbeitenden Muskeln in das Herz, so werden auch mehr Spiritus im Kleinhirn erzeugt und mehr Spiritus zum Herzen herabgeschickt. Dadurch wird die Herztätigkeit bei Körperarbeit beschleunigt, also durch einen Automatismus der Selbstregelung (Cap.100), wie wir heute sagen würden.

In Italien verbindet Giovanni Alfonso Borelli (1680/81) Galileische Physik, Euklidische Geometrie, Korpuskellehre und Anatomie zu einer neuen iatromechanischen Physiologie. Auch er betont wie WiLlis die innere Bewegtheit der Blutteilchen als eigentliche Ursache der Vitalität und der meisten Lebensvorgänge (causam et stimulum motus nempe originem vitae [Cap.111]). Aber die Quelle dieser «motio tremula» ist die Luft, aus der elastische spiralige Partikel mit der Atmung in das Blut gelangen ${ }^{43}$. Ein Feuer im Herzen existiert nicht, wie er durch erstmalige Messung mit dem Thermometer feststellt. Eine Muskelfaser kontrahiert sich, wenn die im Blut befindlichen tartarischen Partikel des Blutes mit einfließenden Partikeln des Nervensaftes zusammentreffen ${ }^{44}$. Dann erfolgt eine fermentative Aufwallung, welche den Hohlraum der Fasern aufbläht und sie dadurch verkürzt. So ist es im Skeletmuskel und so auch im Herzmuskel. Die Eigentätigkeit des Herzens beruht nach Borelli, ähnlich wie bei Lower, auf dem konstanten Zufluß des Herznervensaftes. Aber Borelli geht über LOWER hinaus und versucht erstmals eine Erklärung des alternierenden Wechsels von Systole und Diastole des Herzens ${ }^{45}$. Wie aus einem Glasgefäß mit einem feinen Sprung die Flüssigkeit in einzelnen Tropfen rhythmisch austritt, so kann auch über die schwammig gebauten Herznerven der auslösende Nervensaft nur periodisch einfließen, so daß das Herz in rhythmischem Wechsel «necessitate et automa movetur». Das war seit DESCARTES der erste neue Versuch, die periodische Herzbewegung andersartig, und zwar durch einen vorgeschalteten pulsierenden Mechanismus, zu erklären.

43 Giovanni Alphonso Borelli, De motu animalium, Roma 1680/81, zit. nach Bibliotheca Anatomica, 1685, Tom. II, S. 967.

44 Borelli, ebenda, S. 928.

45 Borelli, ebenda, S. 951/952. 
Ganz ähnlich interpretiert Friedrich Hoffmann ( $†$ 1742), der Hallenser Kliniker, 1703 die Kontraktion des Herzmuskels durch das Einfließen von Blut und expensiven ätherischen Spiritusteilchen über die Nerven ${ }^{46}$ in die Fasern des Herzmuskels (1730/70-85). Die Diastole erfolgt dann durch den Zufluß und den Druck der expansiven ätherischen, elastischen, warmen und bewegten Blutteile auf die sich dehnenden Herzwände ${ }^{47}$. Die Kopplung von Systole und Diastole erklärt er so: Die bewegende Maschine des Herzens ist so konstruiert, daß die Systole der Kammer die Füllung, d. i. die Diastole der Arterien und den Puls hervorruft, das löst als Reaktion die Systole der Arterien aus, und über den Rücklauf des Blutes zum Herzen wird wieder die nächste Diastole erzeugt ${ }^{48}$. "Sic motus fit perpetuus» $(1730 / 70)$. Man sieht, wie man aufgrund neuer Beobachtungen oder neuer Theorien jeweils zu anderen Interpretationen der Herztätigkeit gekommen ist.

Wieder eine andere Erklärung, bei der ebenfalls die Herznerven eine maßgebliche Rolle spielen, findet sich bei dem berühmten Leidener Kliniker Herman BoerhaAve $\left(\dagger 1738\right.$ ) in seinem Lehrbuch ${ }^{49}$ von $1708, \S 185$. Die Herznerven verlaufen nach ihm auf ihrem Weg ins Herz zwischen der Aorta, der Arteria pulmonalis und den Vorhöfen. Damit hängt die Entstehung des Wechsels von Systole und Diastole folgendermaßen zusammen. Wörtlich heißt es (Ausgabe 1780 von J.P.EBerhard, S. 231):

«Aus dieser Lage der Nerven muß man es hauptsächlich herleiten, warum die Zusammenziehung des Herzens die darauf folgende Erweiterung verursache, und warum die Erweiterung wieder eine darauf folgende $\mathrm{Zu}$ sammenziehung nothwendig nach sich ziehe. Es wird nemlich ein Muskel gelähmt, wenn er weder das Schlagaderblut, noch den Nervensaft empfängt. Wird nun das Herz zusammengezogen, so dehnt es die großen Schlagadern aus und drückt die dazwischen liegenden Nerven zusammen. Es treibt aber

46 Fr. Hoffmanni, Fundamenta Medicinae, Halae Magdeburgicae 1746, S. 68, 69, 70, besonders § XVIII.

47 Das Blut füllt die Herzkammern und «calore expansivo diastolem efficit» (HoFFMANnI Fridericir, Medicina Rationalis Systematica, Tom. I, Venetiis 1730, S. 83), ferner Fundamenta Physiologiae, Halae Magdeburgicae 1746, § XVI, S. 68.

48 HoffmanN: "Cordis machina motoria tam affabre constructa est, ut ejus systole diastolen arteriarum, quae pulsus est, producat, et diastole arteriarum rursus provocet earum systolen, quae causa postea rursus diastoles cordis evadit, et sic motus perpetuus. Quam ob rem non inepte perpetuum mobile cor dici potest ...» (ebenda, S. 70).

49. Hermannus BoerhaAve, Institutiones Medicae, Ultima Editio, Parisiis 1747. 
auch zugleich das Blut aus den Kranzschlagadern heraus. Es wird also von eben der Ursache, welche das Zusammenziehen verursachte, gelähmt. Wird das Herz schlaff und weich, so ziehen sich die Schlagadern wieder zusammen, und der Raum zwischen ihnen wird größer, die Nerven werden daher befreit und werden nicht mehr zusammengedrückt, und dieses ist das erste, was zur Bewegung des Herzens erfordert wird (§402.8). Zieht sich ferner die große Schlagader zusammen, so wird sie denjenigen Theil ihres Bluts, der dem Herzen am nächsten ist, in die Kranzschlagadern treiben. Dieses ist das zweite, was zur Bewegung des Herzens erfordert wird (§ 401.) Zu eben der Zeit wird das Herz auch mit dem Blut der Blutadern durch die zusammengezogenen Ohren angefüllt. Hieraus entsteht eine besondere Ursache der Bewegung im Herzen.»

Ganz ähnlich heißt es bei dem BoerhaAve-Schüler und Berliner Arzt Johann Theodor Eller ( $† 1760)$ in seiner Physiologia et Pathologia Medica seu Philosophia Corporis Humani (1757/509). Es handelt sich auch hier um ein mechanomorphes Modell einer Selbststeuerung der alternierenden Herzaktion.

Der Baseler Јон. Rodolphus Stehelin fand dazu 1751 schließlich noch eine elektrische Variante. Da das Elektrisieren den Herzschlag beschleunigt und auch manches andere für eine elektrische Natur des Nervensaftes spricht ${ }^{50}$, so bewirkt das elektrische Fluidum der Herznerven, wenn es ins Blut übergeht, durch seine reizenden Eigenschaften die Systole; hört der Zufluß auf, so tritt Diastole ein. Je mehr elektrisches Fluidum durch die Nerven ins Herz gelangt, desto schneller wird das Herz schlagen.

\section{Albrecht von Haller erklärt die Herzaktion durch die Irritabilität}

Durch Experimente und Überlegungen kam der Schweizer Albrecht voN Haller (†1777), damals in Göttingen, zu der Feststellung, daß die Herznerven fälschlich für die Automatie und den alternierenden Wechsel zwischen Kontraktion und Erschlaffung verantwortlich gemacht würden. Denn auch ein ausgeschnittenes Herz schlägt noch lange weiter. Es ist auch durch vielerlei Arten von Reizen wieder zum Schlagen zu bringen, selbst wenn es schon längere Zeit stillgestanden hat. Man kann das mit Wärme,

50 Joh. Rodolphus Stehelin (Basiliensis), Dissertatio physico-medica de pulsibus, Basel 1749. Gedruckt in Disputationum anatomicarum selectarum, Vol. VII, Ed. AlberTus v. HALleR, Göttingen 1751, S. 345 ff. 
mit Nadelstichen, mit Lufteinblasen usw. erreichen. Jede Muskelfaser vermag auf einen solchen Reiz mit einer Kontraktion zu antworten, das bedeutet: die Muskelfaser besitzt Irritabilität, Reizbarkeit oder, wie wir heute sagen würden, Kontraktilität. Der wirksamste Reiz ist das Blut. Sobald Blut in den Vorhof fällt, reizt es ihn zur Kontraktion und bewirkt die Entleerung in die Kammer. Das von dort ausfließende Blut reizt wieder die Kammermuskeln zur Systole. Sind dann die Herzräume wieder vom Blute befreit, dann hört der Reiz auf und der Muskel erschlafft ${ }^{51}$. So ziehn sich die Herzabteilungen entsprechend den vorwärts wandernden Blutreizen jeweils zusammen. Die Irritabilität oder Muskelkraft HaLlers ist eine spezifisch lebendige Fähigkeit und kommt weder mechanischen noch technischen Gebilden zu (Haller, Elementa, Band I, 5. Abschnitt 1757). Nur wenige Jahre später gelang es dann Felice Fontana (1763), die wahre Ursache der diastolischen Erschlaffung des Herzens festzustellen, nämlich die Refraktärphase ${ }^{52}$. Nach jedem Schlag verliert das Herz für eine gewisse Zeitdauer seine Reizbarkeit und sein Kontraktionsvermögen. Das bedingt seine Periodizität. Sosehr noch Haller in der Tradition der CartesianischBoerhaaveschen Mechanik steht, so bewirkte doch seine Irritabilitätslehre eine Belebung vitalistischer Ideen, in einem Jahrhundert, welches geneigt war, das Lebendige aus anderer Sicht zu betrachten, ihm neue Seiten abzugewinnen und es anders als mechanisch zu interpretieren. Wider Willen gab er den mächtigen Strömungen eines neuen Vitalismus im 18. Jahrhundert Nahrung.

51 Albrecht von Hallen stellt die Kausalkette in seinem Grundriß der Physiologie für Vorlesungen, mit Anmerkungen versehen von Wrisberg, Sömmering und Mecked, Berlin 1788, § 120, folgendermaßen dar: «Allein mir scheint die Natur äußerst einfach zu verfahren, das Ohr wird angefüllt, wenn es schlaff ist, durch die Muskelkraft der nahe liegenden Vene: das Herz wird auf gleiche Weise zusammengezogen, wenn es das Blut, welches das Herzohr hereinbringt, reizt. Es wird, nachdem es das Blut erhalten hat, durch seine Reizbarkeit, und den Reiz selbst, durch den die Fibern zur Zusammenziehung gebracht werden, zugeschnürt, es leert sich vom Blut aus, und nachdem es vom Reiz befreit worden, ruht es, und wird schlaff. Ist's nun schlaff, so wird es durch die Zusammenziehung des Ohrs, welches der nemliche Reiz des venösen Bluts reizt, angefüllt, da die fortwährende Würkung des Herzens und der Arterien beständig Blut ins Herzohr treibt.»

52 Felice Fontana, De legibus irritabilitatis, Lucca 1763. Deutsche Ausgabe von HebenSTREIT, Felix Fontanas Beobachtungen und Versuche über die Natur der thierischen Körper, Leipzig 1785, S. 1-61. 


\section{Der Vitalismus und die Interpretation der Herzaktion}

Mit Georg Ernst Stahl (†1734) in Halle fing der Widerspruch gegen den herrschenden Mechanismus an. In seiner Streitschrift gegen LeIBNIz heißt es (1720): Die Körpermaschine besitze aus sich selbst keine Aktivität, keine Intention, vielmehr seien alle Teile passiv. Jede Bewegung wird den Teilen unmittelbar von der Seele mitgeteilt, deren Bewegtheit oder motio sich auf die Organe überträgt. Dabei ist sie allerdings von der Beweglichkeit und Eigenart der Teile abhängig. Aber die Seele allein hat das energetische Vermögen, etwas in Bewegung zu setzen. Sie macht das direkt, ohne spezifische facultates und ohne besondere spiritus ${ }^{53}$. Für einen solchen Psychovitalismus stellen sich neue Fragen nach den Wirkungszusammenhängen unter den Herzteilen nicht. Wenn Albrecht von Haller auch absolut ein StaнL-Gegner war, so wirkte doch seine Irritabilitätslehre zugunsten der Annahme spezifisch vitaler Kräfte. Wir finden sie als vis essentialis bei C.Fr. Wolf, als Bildungskraft bei Blumenbach, als forces animées bei Sauvages in Montpellier, als Lebenskraft bei Hufeland, JohanNes Müller, Liebig u.a. Aber bei J. Müller (†1856) ist diese organische Kraft nicht selbsttätig, sondern das Räderwerk des Organismus bedarf dazu dauernd äußerer Impulse, der Reize. «So unpassend der Vergleich mit einem Mechanismus auch sein mag, die organische Kraft, welche in den organischen Körpern den zum Leben notwendigen Mechanismus erschafft, ist doch keiner Akte ohne diesen äußeren Impuls ... fähig ${ }^{54}$.» So muß auch die Herzaktion auf Reizen beruhen, sie muß in der Wechselwirkung des Blutes oder der Nerven mit der Herzsubstanz liegen; diese Frage hat MüLLER zur Zeit für unlösbar gehalten ${ }^{55}$.

53 Georg Ernst Stahl, Theoria Medica Vera, Halle 1708, S. 491; auch S. 567; ferner in Negotium otiosum, Halae 1720, siehe dazu K.Е. RотнsснUн, Leibniz, die prästabilierte Harmonie und die Ärzte seiner Zeit, Akten des Internationalen Leibniz-Kongresses, Hannover 1966, Band II, S. 231-254, besonders S. 238-240.

54 Jон. Müller, Handbuch der Physiologie des Menschen, erster Band, Coblenz 1833, S. 177.

55 Johannes MüLler äußert sich über die Ursache des Herzschlages und der rhythmischen Folge von Vorhof- und Kammerkontraktion folgendermaßen:

«Da das Herz nun gleich allen Muskeln durch Reize zur Contraction angeregt wird, so liegt es sehr nahe anzunehmen, daß das Blut der Herzhöhlen selbst das Herz zu Contractionen reizt ... So nothwendig indessen eine gewisse Blutmenge und eine gewisse Anfüllung der Herzhöhlen zur Unterhaltung der Thätigkeit des Herzens ist, und so gewiß jede mechanische Ausdehnung des Herzens von innen Zusammenziehung in ihm hervorrufen muß, so ist der Reiz des Blutes in den Herzhöhlen doch nicht der letzte Grund der rhythmischen Zusammenziehung des Herzens. Denn auch das blutleere 


\section{Fortschritte durch die experimentelle Herz-Physiologie der letzten hundert Jahre}

Es ist nicht möglich, alle weiteren Einzelschritte zu schildern, welche zu unseren heutigen Vorstellungen von Automatie, Koordination und Regelung der Herztätigkeit geführt haben. Wesentlich war die Feststellung von Stannius (1852), daß es eine bestimmte Stelle im Herzen gibt, von der der Anstoß zur Herzaktion ausgeht, nämlich die Sinusregion ${ }^{56}$. Die Automatie des Herzens hat also einen festen Ort. Aber was spielt sich hier ab ? Das haben erst die elektrophysiologischen Untersuchungen der letzten zwanzig Jahre geklärt. In der Sinusregion erfolgen rhythmische elektrische Entladungen, als Aktionspotential sichtbar und meßbar. Demnach besitzt das Herz nicht nur Reaktivität gegenüber einem Reiz, wie noch HALler oder MüLlER annahmen, sondern Autoaktivität in Form einer Entladung von Grenzflächen analog von Kippschwingungen. Wie wird nun die Aufeinanderfolge der Vorhöfe und Kammern gesteuert? Durch einen beim Warmblüterherzen anatomisch vorgebildeten Weg der Erregungsausbreitung über das His'sche Bündel und die Purkinjefäden. Dadurch und durch die Refraktärphase ist die zeitliche und rechtläufige Aufeinanderfolge der Vorhof- und Kammerkontraktionen bedingt. Was die Regelung der Herztätigkeit anbelangt, so hat sich auf der einen Seite tatsächlich eine Wirkung der Herznerven nachweisen lassen ${ }^{57}$. Aber auch unabhängig davon sind die

Herz setzt seine Contractionen noch schwächer fort ... Nun könnte man zwar diese von dem Reiz der Luft ableiten, und an jenes Gesetz erinnern, daß wenn ein Reiz auch beständig ist, die Contractionen doch oft nur periodisch erfolgen können. Allein dasselbe geschieht im luftleeren Raum, und ohne einen inneren Grund könnte sich nicht die regelmäßige Aufeinanderfolge der Ventricular-Contraction auf die Contraction der Vorhöfe erhalten. Die Ursache muß also viel tiefer liegen. Es muß in der Organisation des Herzens und in der beständigen Wechselwirkung des Blutes in den kleinsten Gefäßen mit der Herzsubstanz oder in der Wechselwirkung der Herznerven und der Herzsubstanz etwas liegen, was entweder anhaltend wirkt, worauf aber das Herz nur periodisch reagiert, oder das selbst periodisch auf das Herz einwirkt. Die Lösung dieser Frage ist unendlich schwierig, bei dem jetzigen Standpunkt der Wissenschaft unmöglich» (ebenda, S. 177).

${ }^{56}$ H.Stannius, Zwei Reihen physiologischer Versuche, Versuche am Froschherzen, Müllers Arch. Anat. Physiol. wiss. Med., 1852, S. 85-92.

${ }^{57}$ Die erste wirklich erfolgreiche Reizung des N. vagus mit Verlangsamung der Herzfrequenz gelang Ernst Hnr. Weber und Eduard Weber 1846. Wichtige Beiträge dazu lieferte Albert v. Bezold 1863, vgl. dazu R. Herrlinger und I. Krupp, Albert von Bezold (1836-1868), Ein Pionier der Kardiologie, Stuttgart 1964. 
Arbeitsräume des Herzens in der Lage, auf vermehrte Füllung mit erhöhtem Auswurfvolumen und auf erhöhten Entleerungswiderstand mit erhöhter Druckentwicklung zu reagieren. Das besagt das Starlingssche Gesetz der Herzarbeit ${ }^{58}$ (1915). Damit ist die Herzforschung wieder ein wesentliches Stück weitergelangt zur Erkenntnis der sogenannten «Selbststeuerung » der Herzaktion. Damit wollen wir die Schilderung der Details beenden.

\section{Schlu $\beta$}

Die Darstellung mußte die 2000 jährige Geschichte der Herzinterpretation in großen Schritten durchmessen. Wie man sieht, hat man zu allen Zeiten von sehr unterschiedlichen Voraussetzungen her die Tätigkeit und Bedeutung des Herzens zu verstehen versucht, oft tastend, ja hoffnungslos. Wie schrieb doch Harvey 1628 zu Eingang seines denkwürdigen Buches ${ }^{59}$ : «Als ich mein Sinnen und Trachten zum ersten Male der Beobachtung des lebenden Herzens zuwandte, da fand ich die Sache so voller Schwierigkeiten, daß ich ... meinte, die Bewegung des Herzens sei nur Gott bekannt » (Cap.1). Aber de facto hat er mit der Kreislauflehre einen entscheidenden Schritt in die Zukunft getan. Zuversichtlicher ist HALLER in einem seiner Gedichte des Jahres 1733: «Bald suchst Du in der Wunder-Uhr, dem Meisterstücke der Natur, bewegt von selbstgespannten Federn, Du siehst des Herzens Unruh gehen, Du kennst ihr Eilen und ihr Stehen und die Vernutzung (= Abnutzung) an den Rädern. ${ }^{60}$ Heute endlich glauben wir die Autoaktivität, Reaktivität, Regelung und Steuerung des Herzens so weit zu durchschauen, daß wir versuchen können, ein «künstliches Herz» zu bauen. Und damit stehen wir wieder am Ausgangspunkt unserer Überlegungen. Aus der Kenntnis der Arbeitsweise des Herzens und der sich dabei vollziehenden Teilprozesse gewinnen wir die Möglichkeit, die Lebensvorgänge zu manipulieren, die Herzaktion in anderem Material, aber mit gleichartiger Leistung nachzuahmen, und damit einen Herzersatz anzustreben. Darin steckt die Denkarbeit von mehr als 2000 Jahren.

58 Ernest Starling, Lecture on the law of the heart, Cambridge 1915.

59 William Harvey, Exercitatio anatomica de motu cordis et sanguinis, Francoforti 1628.

60 Albrecht von Haller, Gedichte, Ed. Ludwig Hirzel, Frauenfeld 1882, S. 114, Vers 103-108. 